\title{
Ecosistema con creatividad, investigación e innovación basado en las competencias transversales frente a las exigencias profesionales del siglo XXI
}

\section{Ecosystem with creativity, research and innovation based on transversal competences facing the professional demands of the 21 st century}

\author{
Violeta L. Romero Carrión, ${ }^{\text {a }}$ Segundo A. García Flores² y José M. Palacios Sánchez ${ }^{3}$ \\ Universidad Nacional Federico Villarreal. Lima, Perú ${ }^{123}$
}

iD ORCID ID: https://orcid.org/0000-0003-3260-4776 ${ }^{1}$

ORCID ID: https://orcid.org/0000-0001-8587-32112

(iD) ORCID ID: https://orcid.org/0000-0002-1267-5203 3

Recibido: 15 de agosto de 2020

Aceptado: 15 de noviembre de 2020

\section{Resumen}

El objetivo de la investigación fue determinar si el Ecosistema con creatividad, investigación e innovación, basado en las competencias transversales, responde a las exigencias profesionales del siglo XXI. Para el estudio se aplicó el cuestionario de competencias transversales, del Proyecto Tuning, dirigido a 30 estudiantes universitarios, en cuyo caso se indagó sobre el logro en su formación, mientras que, en el caso de los 30 empleadores, fue conocer la importancia que le asignan a las competencias que deben poseer los nuevos profesionales. Luego de probar la normalidad de la distribución de los datos, se aplicó el estadístico de prueba de hipótesis T de Student para muestras independientes, obteniendo un valor altamente significativo, con respecto a que las competencias comprendidas en el Ecosistema con creatividad, investigación e innovación, son distintas, entre lo logrado por los estudiantes y lo exigido a los nuevos profesionales por los empleadores de las organizaciones del siglo XXI. Se recomienda disminuir la brecha entre las competencias transversales, logradas por los universitarios y las exigidas por los empleadores, mediante la actualización frecuente del currículo de estudio acorde a las demandas del mercado laboral. 
Palabras clave: creatividad, investigación, innovación, competencias transversales, mercado laboral, empleadores.

\section{Abstract}

The objective of the research was to determine if the Ecosystem with creativity, research and innovation, based on transversal competences, responds to the professional demands of the 21st century. For the study, the questionnaire of transversal competences, of the Tuning Project, was applied, aimed at 30 university students, in which case the achievement in their training was investigated, while, in the case of the 30 employers, it was to know the importance of assigned to the competencies that new professionals should possess. After testing the normality of the data distribution, the Student's t hypothesis test statistic was applied for independent samples, obtaining a highly significant value, with respect to the fact that the competences included in the Ecosystem with creativity, research and innovation, They are different, between what has been achieved by students and what is demanded of new professionals by the employers of 21 st century organizations. It is recommended to reduce the gap between transversal skills, achieved by university students and those required by employers, through frequent updating of the study curriculum according to the demands of the labor market.

Keywords: creativity, research, innovation, transversal competences, working market, employers.

\section{Introducción}

El escenario del siglo XXI presenta un mundo globalizado, con información desbordante, digitalizado, amplio acceso a la comunicación y muy cambiante. En este contexto, en la educación superior se ve la necesidad de realizar cambios para atender las exigencias de este nuevo panorama, caracterizado también por la incertidumbre. Concretamente, hay incertidumbre en relación a las innovaciones disruptivas que debemos impulsar mediante políticas públicas, para producir la satisfacción de necesidades (Bogers, Chesbrough y Moedas, 2018). Según Ordóñez (2010) la creatividad es una alternativa para afrontarlo de manera novedosa y también la innovación, que posibilita su implementación, cabe añadir, que estos elementos se vinculan a su vez con la investigación.

\section{Creatividad e Innovación}

En esencia el ser humano tiene las capacidades de creación e innovación. Ambas mantienen una relación muy estrecha, pero a la vez diferenciada. La generación de ideas a través de un proceso mental, es ser creativo mientras que la aplicación práctica y útil de las ideas resulta ser innovador (Iglesias y Rodicio, 2013). Es decir, "En la creación, el vértice es la generación de ideas, y en la 
innovación, dicho vértice es la implementación de las ideas” (Hernández, Alvarado y Luna, 2015, p. 138).

En la sociedad digital, ser innovador implica tener cualidades y habilidades que permiten combinar ideas, relacionarlas, asociar conceptos e integrarlos en un todo útil, que lleva a un producto único novedoso. Ser creativo es producir ideas originales, novedosas, espontáneas, de relevancia, que permitan transformar un hecho o situación de la realidad. "La innovación es una competencia susceptible de aprenderse y practicarse” (Hernández, et al. 2015, p. 140). Así, la universidad debe repensarse, responder a las preguntas de los estudiantes ¿para qué voy a la universidad? ¿para ser creativo?; el reto es indagar sobre la forma de desarrollar la innovación en la formación profesional asumiendo que el principal activo es el conocimiento.

La Teoría de J. Guilford, desarrollada con sus colaboradores desde 1950 en la universidad de California del Sur, considera que existen dos estilos de pensamiento: convergente y divergente. El pensamiento convergente es secuencial, ordenado, se desplaza en una dirección establecida, permite llegar a conclusiones lógicas e intenta llegar a la respuesta correcta; en cambio el pensamiento divergente implica mirar desde distintas perspectivas, procura más de una respuesta, rompe con esquemas rígidos, no se basa en suposiciones únicas y previas, establecer reformas sobre lo que parece insólito o inútil, transitar por caminos impensados, investigar para producir algo novedoso, en síntesis está más relacionado con la creatividad.

La Teoría de Edward De Bono del año 1967, considera la existencia del pensamiento lógico, racional, convencional o vertical, en el que recorremos senderos ya transitados, usamos experiencias y situaciones afines, pero, ese pensamiento lógico (fundamentalmente hipotético deductivo) pierde su utilidad cuando se procura soluciones a problemas nuevos. La mente desarrolla modelos clásicos de conceptos, lo que impide el uso de la nueva información existente, salvo que surja algún medio de reestructurar los modelos vigentes, con nuevos datos, volviendo a mirar, para incorporar nuevas soluciones, creativas, acorde a la necesidad, ese sería en síntesis el objetivo del pensamiento lateral. El foco central de esta teoría, está en observar los retos desde un ángulo diferente, donde urge el desarrollo de competencias transversales desde la formación inicial universitaria.

Complementa a la creatividad y la innovación; la investigación científica, definida como un proceso complejo, sistemático, de resolución de interrogantes y producción de conocimientos, que tiene reglas propias, las cuales prescriben cómo se asegura que el proceso de indagación 
científica satisface la definición de la verdad y los mecanismos de transferencia de ella (Piscoya, 1987 y Navarro et al., 2017). Esto posibilita la concepción de un Ecosistema, en el que interactúan la creatividad, la investigación y la innovación, enmarcados en el conocimiento, lo cual se consolida bajo el enfoque sistémico desde la perspectiva de Edgar Morín, analizado por Pereira (2010) cuando refiere "la interconexión y las interacciones entre los objetos, las personas y el ambiente como un todo, lo cual sirve de fundamento para la propuesta educativa, en la que el conocimiento se debe abordar de manera integral y no fragmentada" (p.67). Las partes (creatividad, investigación e innovación) están vinculadas, pero también son interdependientes entre sí, resultando que el todo (conocimiento científico) es mayor que la suma de sus partes.

\section{Competencias Transversales o genéricas}

Según la Organización para la Cooperación y el Desarrollo Económico (OCDE), las competencias son "el conjunto de conocimientos, atributos y capacidades que pueden aprenderse y permiten a los individuos llevar a cabo con éxito y en forma consistente una actividad o tarea, y que pueden desarrollarse y ampliarse a través del aprendizaje” (OCDE, 2012, p. 12). Esto involucra la movilización combinada de habilidades, conocimientos, valores, emociones y otros componentes sociales para un accionar efectivo en una situación considerada compleja. Ciertamente, competencias transversales o genéricas como "la resolución de problemas complejos, el pensamiento crítico, el trabajo en equipo, la resiliencia y la adaptabilidad" OCDE, 2019, p.28), incorporan de manera efectiva aptitudes, conocimientos y valores que son necesarios para el éxito laboral y que son compartidas por diferentes especialidades.

Para Rodríguez (2012), las competencias transversales o genéricas constituyen una de las características que influyen directamente en el nivel de empleabilidad y de retribución en las organizaciones, donde se vinculan el uso de herramientas tecnológicas, interacción con los recursos humanos, manejo de idiomas, capacidad de negociación y otros; la adquisición de competencias se entiende como "un proceso dinámico que requiere de variedad de acciones sistematizadas, integradas y paralelas al currículo universitario para obtener el máximo rendimiento" (Martínez \& González, 2019, p.20); además el nivel de competencias constituye un diferenciador crítico del rendimiento (Bustamante, Oyarzún, Grandón y Abarza, 2015).

En esa línea de ideas, los cambios socioeconómicos son complejos y se reflejan en las distintas exigencias laborales de las organizaciones. Por ello, no es suficiente que los egresados 
sean especialistas en un área determinada y tengan una formación meramente técnica o de conocimiento, sino que es necesario que evidencien un desarrollo personal y social para tener una visión integral al abordar los problemas. Consecuentemente la universidad debe integrar estos aspectos en el currículo de estudios con periodicidad frecuente.

El proyecto Tuning, se refiere a la competencia como "Una combinación dinámica de atributos, en relación a procedimientos, habilidades, actitudes y responsabilidades, que describen los encargados del aprendizaje de un programa educativo o lo que los alumnos son capaces de demostrar al final de un proceso educativo" (Lafontaine, 2010, p.9). Con posterioridad a su publicación y difusión en América Latina, se han venido incorporando a las políticas educativas que promueven el aseguramiento de la calidad de la educación. Al respecto, un estudio de Llaque (2020) analiza cinco retos que debe afrontar la gestión de la educación superior, que van desde la incorporación del factor productivo, las TICs, perfiles competenciales, enseñanza centrada en el estudiante e internacionalización.

Pedroza (2018) en tono prospectivo, se refiere a la universidad como aquella que tendrá currículo inteligente y que dicho currículo estará caracterizado por la conectividad entre redes virtuales; marcado por "los cambios en el diseño, estructura e itinerarios curriculares a partir de la informatización" (p.186). Lo cual confirma la pérdida de vigencia de los currículos de estudio, dado el avance tecnológico y de la virtualidad, lo cual no podemos ignorar en el campo educativo.

Mayorga-Fernández, Madrid-Vivar \& Padial-Díaz (2016) encontraron que el 57,87\% de las competencias profesionales exigidas en el mercado laboral, no se encuentran recogidas en las competencias formativas y tampoco las competencias transversales que son las más requeridas a los profesionales. De igual forma el estudio de Álvares (2016) concluye que existe una desarticulación entre la formación universitaria y las competencias para un desempeño exitoso de los egresados. Es decir, las competencias solicitadas por los empleadores no son prioridad en la formación curricular.

En este contexto consideramos el Ecosistema con creatividad, investigación e innovación (E-cii), como una unidad de organismos interdependientes, y su operacionalización requiere de las competencias transversales, y su logro, deberá responder a lo requerido por los empleadores del siglo XXI. Consecuentemente, planteamos el siguiente objetivo: Determinar si el Ecosistema con creatividad, investigación e innovación responde las exigencias profesionales del siglo XXI 
a partir de las competencias transversales. Específicamente en 1). caracterizar el ecosistema con creatividad, investigación e innovación que responde a las exigencias profesionales del siglo XXI a partir de las competencias transversales; 2). determinar las competencias exigidas por los empleadores a los profesionales del siglo XXI en el contexto de las competencias transversales.

\section{Materiales y método}

Esta investigación es un estudio de caso, para una colectividad universitaria y los empleadores de organizaciones vinculadas a esta, en la que se considera una variable nominal desde dos perspectivas.

Ámbito espacial y temporal: Universidad Nacional de Lima, año 2019.

Tipo: Investigación no experimental, expo facto y transversal.

Nivel: descriptivo correlacional.

Diseño:

$\mathrm{O}_{1}$
$\mathbf{r}$
$\mathrm{O}_{2}$

$$
\begin{aligned}
\mathrm{M} & =\text { Competencias del Ecosistema } \\
\mathrm{O}_{1} & =\text { Observación }- \text { contexto universitario } \\
\mathrm{O}_{2} & =\text { Observación }- \text { contexto laboral } \\
\mathrm{r} & =\text { relación }
\end{aligned}
$$

Población: estudiantes de las diversas facultades de la Universidad Nacional Federico Villarreal (UNFV) en el año 2019 y empleadores de organizaciones en la cual los estudiantes realizan las prácticas pre profesionales.

Muestra: por conveniencia, debido a la accesibilidad de contar con estudiantes de las diversas facultades, quienes asistían a un curso formativo de Investigación, organizado por áreas: Ingenierías, Ciencias de la Salud y Básicas, Ciencias Sociales, Humanidades y Empresas. Los empleadores fueron de las diversas empresas y organizaciones donde los estudiantes realizaban las prácticas pre profesionales, siendo 30 estudiantes y 30 empleadores.

Instrumento: Cuestionario de 30 preguntas basado en las Competencias Genéricas, identificadas por el proyecto Tuning.

Fiabilidad y Validez: Las propiedades métricas del cuestionario fueron realizadas por David Aguado y su equipo - Universidad Autónoma de Madrid (2017). Sin embargo, para corroborar dicha fiabilidad aplicamos el cuestionario a un grupo piloto, en el que el alfa de Cronbach resultó $\alpha=0,944$ lo que demuestra una fuerte relación entre las preguntas. 


\section{Procedimiento}

- Se identificaron las competencias relacionadas con la creatividad, investigación e innovación, contenidas en el cuestionario del Proyecto Tuning.

- Búsqueda en diversas fuentes como periódicos, avisos en páginas virtuales, etc. de los requerimientos competenciales a los nuevos profesionales solicitados por las empresas.

- Se realizó la aplicación del cuestionario, con consentimiento informado, para el manejo bioético de los datos, tanto a nivel de estudiantes como de empleadores.

- El cuestionario aplicado a los estudiantes y empleadores fue el mismo, sin embargo, la diferencia estuvo en la instrucción dada. A los estudiantes se les solicitó que registren el nivel de desarrollo de cada competencia en la universidad; mientras tanto en el caso de los empleadores, se solicitó que indiquen la importancia que le asignan a cada competencia.

- Análisis de los datos para la aplicación del estadístico correspondiente.

- Se estableció la normalidad de los datos recogidos, y consecuentemente se seleccionó el estadístico de prueba de hipótesis T de Student para muestras independientes.

- Comparación de los resultados con la de otros autores y conclusiones.

\section{Resultados}

\section{Caracterización del Ecosistema con creatividad, investigación e innovación}

El Ecosistema con creatividad, investigación e innovación está constituido por tres constructos o conceptos no tangibles, de los cuales inferimos su existencia por sus efectos; conforman un sistema complejo, e interactúan simbióticamente en el hábitat del conocimiento. Cada uno de los subsistemas, creatividad, investigación e innovación son relevantes y al interactuar dentro del sistema complejo del conocimiento científico, posibilitan la creación de nuevos conocimientos, su secuencia y direccionalidad no es necesariamente lineal, pueden interactuar indistintamente (ver figura 1). 


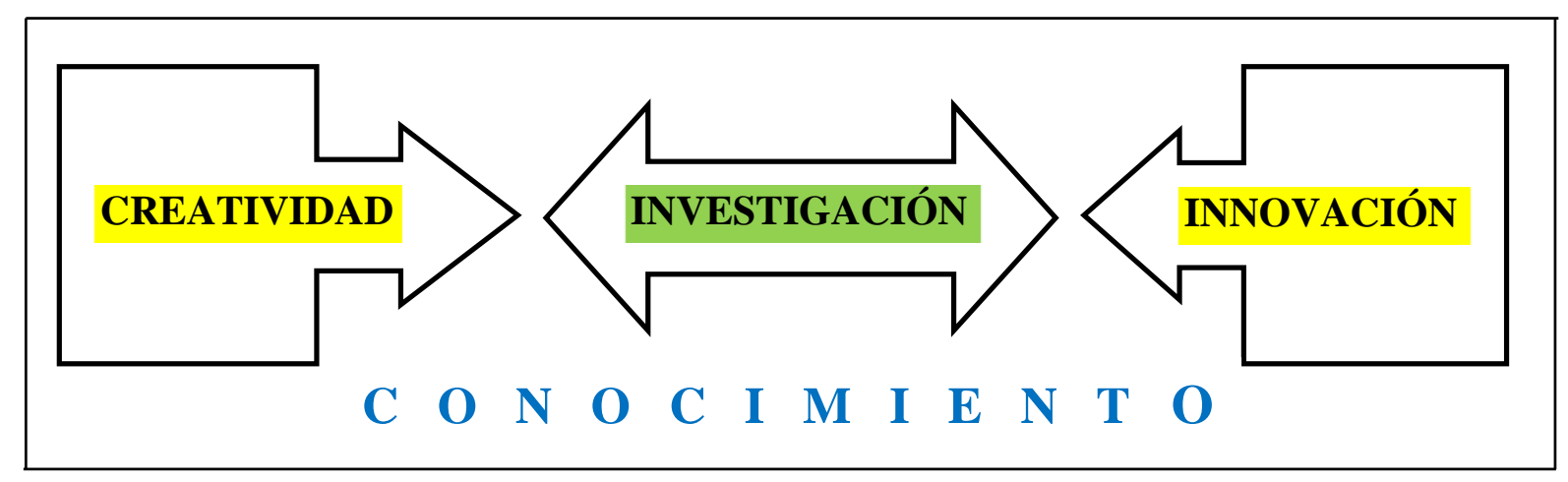

Figura 1. Ecosistema con creatividad, investigación e innovación.

Fuente: elaboración propia.

\section{Competencias transversales requeridas por los empleadores del siglo $X X I$}

Contar con las competencias genéricas o transversales, implica poseer las potencialidades para un accionar efectivo en escenarios diversos y cambiantes, integrando conocimientos, habilidades, motivación y valores. Las exigencias profesionales, provienen desde las organizaciones y la sociedad, la responsabilidad de cumplir con dichas exigencias, se le atribuye en gran medida a la universidad, que deberá incluirlas en los currículos de estudio.

A partir de diversas fuentes como, perfiles profesionales de los últimos tres años, convocatorias para puestos de trabajo de empresas y organizaciones públicas y privadas, avisos de empleo en periódicos, páginas virtuales y redes sociales, se ha elaborado la Tabla 1, con las características profesionales de diversas especialidades. Cabe señalar que las cuales se encuentran incluidas en un $94 \%$, en el cuestionario de Competencias genéricas identificadas por el Proyecto Tuning. 
Tabla 1

Competencias demandadas por los empleadores de las organizaciones del siglo XXI

\begin{tabular}{lll}
\hline & \multicolumn{1}{c}{ COMPETENCIAS } & \\
\hline Analítico & Curioso & Iniciativa \\
Arriesgado & Desarrollo sistémico & Innovador \\
Autónomo & Disposición & Investigador \\
Colaborador & Espontaneidad & Líder \\
Comprometido & Ético & Organizado \\
Comunicativo & Flexibilidad & Originalidad \\
Creativo & Habilidad con la tecnología & Proactivo \\
Crítico & Habilidad con los idiomas & Trabajo en equipo \\
\hline
\end{tabular}

Fuente: Elaboración propia.

En la Figura 2 en base a las 30 preguntas del cuestionario, se registran las cuatro puntuaciones más bajas y también las más altas, desde la apreciación de los estudiantes. Se observa que lo menos logrado es la comunicación en un segundo idioma, mientras que la más lograda, es el respeto a la multiculturalidad. 


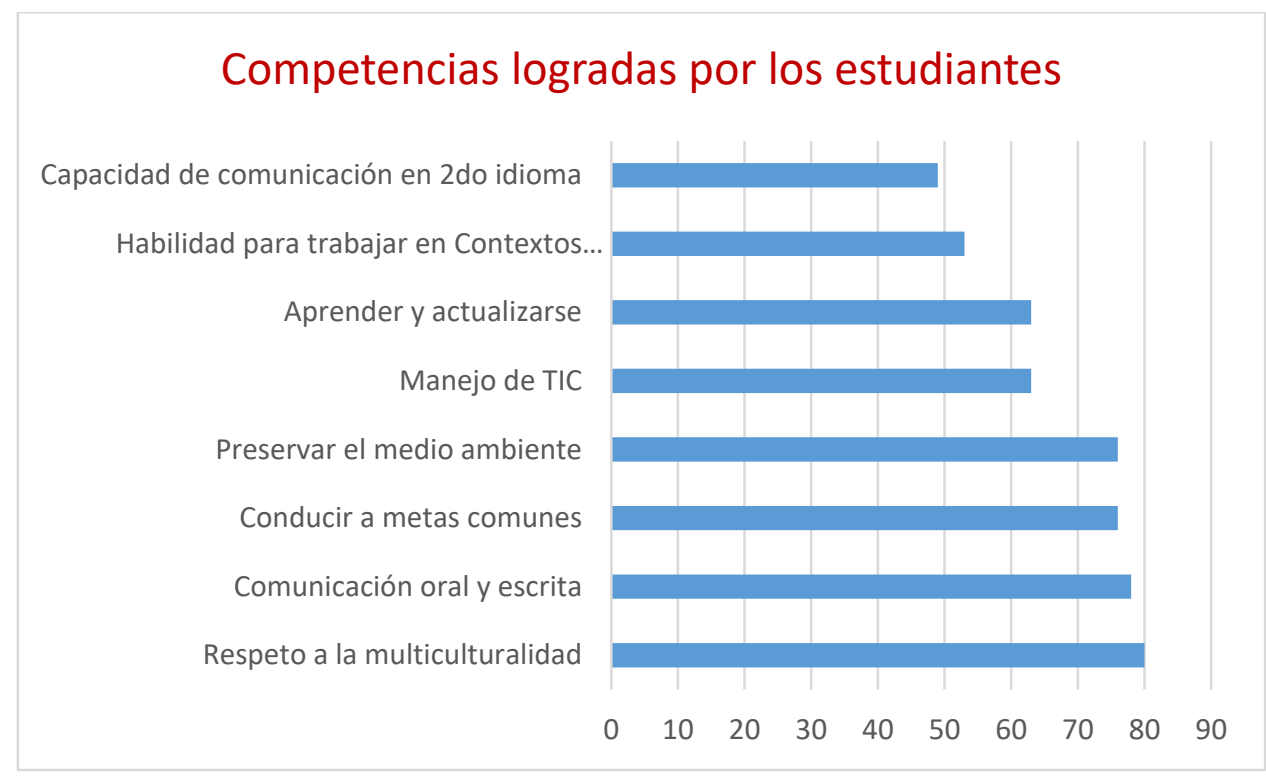

Figura 2. Competencias con el nivel desarrollado en la universidad. Fuente: Elaboración propia.

En la figura 3 se muestra, desde la perspectiva de los empleadores, que la menor puntuación corresponde a la capacidad de comunicación en un segundo idioma, lo cual coincide con la apreciación de los estudiantes, y lo que más puntúan los empleadores es que los jóvenes profesionales realicen la búsqueda de más de una respuesta ante un problema.

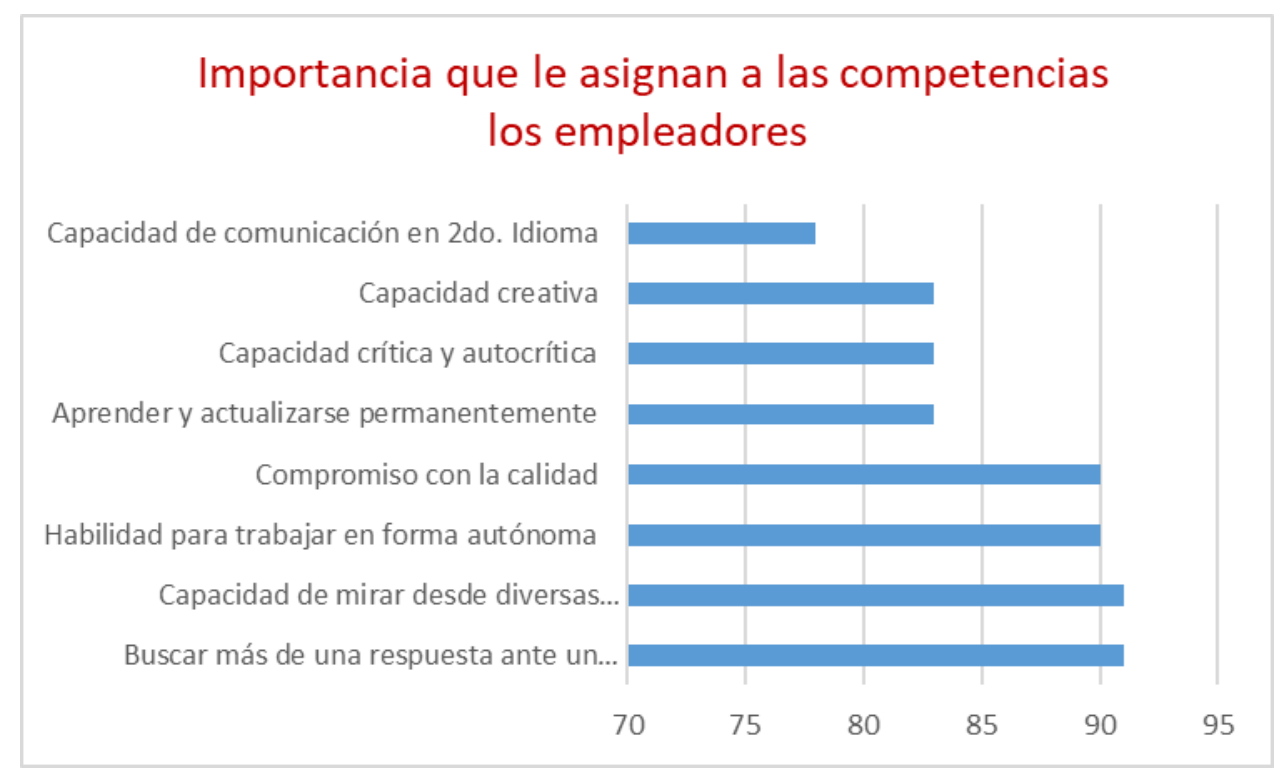

Figura 3. Importancia que le asignan los empleadores a cada competencia. 
Para determinar si las competencias del Ecosistema con creatividad, investigación e innovación, adquiridas por los estudiantes universitarios, difieren, de las esperadas por los empleadores de los nuevos profesionales, se procedió en primer lugar a comprobar la normalidad de la distribución de los datos obtenidos, tanto para los universitarios, como para los empleadores.

Por otro lado, Se observa en la Tabla 2, la significancia mostrada mediante Shapiro-Wilk p $=0,342$ y al ser mayor que 0,05 no se puede rechazar la hipótesis nula, consecuentemente, la distribución es normal en ambos casos, lo que permite la aplicación de las pruebas estadísticas paramétricas.

\section{Tabla 2}

Pruebas de normalidad

\begin{tabular}{lr|r|r|r|r|r|} 
& \multicolumn{3}{c}{ Kolmogorov-Smirnova } & \multicolumn{3}{c}{ Shapiro-Wilk } \\
& Estadístico & gl & \multicolumn{1}{c}{ Sig. } & Estadístico & gl & Sig. \\
\hline Universitarios &, 093 & 30 &, $200^{*}$ &, 962 & 30 &, $\mathbf{3 4 2}$ \\
\hline Empleadores &, 104 & 30 &, $200^{*}$ &, 962 & 30 &, $\mathbf{3 4 2}$ \\
\hline
\end{tabular}

*. Esto es un límite inferior de la significación verdadera.

a. Corrección de significación de Lilliefors

Fuente: Elaboración propia.

\section{Contrastación de la hipótesis}

Se tienen las competencias transversales comprendidas en el Ecosistema con creatividad, investigación e innovación, logradas por los estudiantes de la UNFV, frente a las mismas competencias, exigidas por los empleadores a los nuevos profesionales. Habiendo comprobado la normalidad para ambas muestras, se aplica el estadístico $\mathrm{T}$ de Student para muestras independientes.

$\mathrm{H}_{0}=$ Las competencias del ecosistema-cii son iguales, al logrado por los estudiantes y lo exigido a los profesionales por los empleadores.

$\mathrm{H}_{1}=$ Las competencias del ecosistema-cii son distintas, al logrado por los estudiantes y lo exigido a los profesionales por los empleadores.

En la Tabla 3 sobre la Prueba de T Student de muestras independientes, se muestra con un nivel de confianza del 95\% que la significancia o "p" valor es $p=0,000$. Resultando $p<0,005$ 
entonces rechazamos la hipótesis nula y se determina que las competencias comprendidas en el Ecosistema con creatividad, investigación e innovación, son distintas, entre lo logrado por los estudiantes de la UNFV y lo exigido a los profesionales por los empleadores de las organizaciones del siglo XXI.

\section{Tabla 3}

Prueba de hipótesis para las competencias del ecosistema logradas por los estudiantes y exigidas por los empleadores en muestras independientes

\begin{tabular}{|c|c|c|c|c|c|c|c|c|c|c|}
\hline & & \multicolumn{2}{|c|}{$\begin{array}{l}\text { Prueba de } \\
\text { Levene de } \\
\text { igualdad de } \\
\text { varianzas }\end{array}$} & \multicolumn{7}{|c|}{ Prueba t para la igualdad de medias } \\
\hline & & & & & & Sig. & $\begin{array}{c}\text { Diferencia } \\
\text { de }\end{array}$ & $\begin{array}{r}\text { Diferencia } \\
\text { de error }\end{array}$ & $\begin{array}{r}95 \% \text { de ir } \\
\text { confianza de }\end{array}$ & $\begin{array}{l}\text { avalo de } \\
\text { a diferencia }\end{array}$ \\
\hline & & $\mathrm{F}$ & Sig. & $\mathrm{t}$ & gl & (bilateral) & medias & estándar & Inferior & Superior \\
\hline \multirow{2}{*}{ 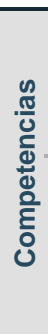 } & $\begin{array}{l}\text { Se asumen } \\
\text { varianzas } \\
\text { iguales }\end{array}$ & 8,948 & ,004 & $-11,27$ & 58 & , 0 & $-17,733$ & 1,573 & $-20,881$ & $-14,585$ \\
\hline & $\begin{array}{l}\text { No se } \\
\text { asumen } \\
\text { varianzas } \\
\text { iguales }\end{array}$ & & & 11,27 & 43,4 & ,000 & $-17,733$ & 1,573 & $-20,904$ & $-14,563$ \\
\hline
\end{tabular}

Fuente: Elaboración propia

\section{Discusión}

Se entiende que los currículos de estudio, son elaborados tomando en cuenta el perfil laboral de los profesionales, que demandan las organizaciones y la sociedad actual, sin embargo, se ha demostrado en esta investigación, que hay diferencias entre las competencias alcanzadas por los universitarios y las demandadas por los empleadores a los nuevos profesionales, esta desarticulación implica muy bajas tasas de empleabilidad entre los egresados de acuerdo con Álvares (2016). Asimismo, coincidimos con Pedroza (2018) cuando manifiesta la necesidad de cambios curriculares con influencia de la informática para afrontar el futuro de la universidad.

Asimismo, observamos que los estudiantes han puntuado con valores bajos, muchas de las competencias transversales recogidas en el cuestionario, referidas a la creatividad, investigación e innovación, lo cual concuerda con lo encontrado por Mayorga-Fernández, et al (2016) quienes 
señalan que, lo exigido por el mercado laboral en cuanto a las competencias profesionales sólo se hallan recogidas en un $42.13 \%$ y tampoco se encuentran las competencias transversales que son las más requeridas a los jóvenes profesionales.

Para satisfacer las demandas laborales de las organizaciones con efectividad, es necesario que se formen a los universitarios con competencias comunicativas en más de un idioma, que sepan argumentar, inferir, deducir y buscar más de una respuesta ante un problema. En tal sentido coincidimos con Hernández, et al (2015) cuando manifiestan, que es necesario un cambio y que sea con soporte ético.

El logro de las competencias específicas de la carrera profesional y de las competencias genéricas, común a todas las carreras, posibilita una mejor vinculación entre la Universidad formadora de profesionales, y la sociedad a través de las organizaciones empleadoras de dichos profesionales; en ese orden de ideas concordamos con Rodríguez (2012), cuando manifiesta que las competencias transversales, influyen directamente en el nivel de empleabilidad y de retribución, que en última instancia es lo que aspiramos que alcancen nuestros egresados, un empleo y adecuada retribución económica.

Por ello, es pertinente manifestar que este estudio de casos, realizado con rigor, pero limitado en cuanto al número de participantes, fundamentalmente por razones de financiamiento, deberá replicarse en la misma universidad y otras, tanto públicas como privadas, para posibilitar resultados y conclusiones de mayor robustez y generalizables.

\section{Conclusiones}

En este estudio, se demuestra con un nivel altamente significativo $(\mathrm{p}=0,000)$ del estadístico de prueba que, las competencias transversales inmersas en el Ecosistema con creatividad, investigación e innovación, son distintas, entre lo logrado por los estudiantes de la UNFV y lo exigido a los profesionales por los empleadores de las organizaciones del siglo XXI. Asimismo, se caracterizó al Ecosistema con creatividad, investigación e innovación, formado por los tres constructos que constituyen un sistema complejo, en el que interactúan para beneficiarse mutuamente, teniendo como hábitat el conocimiento.

Por tanto, Las organizaciones demandan profesionales de diversas especialidades, pero todas concuerdan en las exigencias de las competencias transversales, principalmente como ser colaborador, creativo, innovador, flexible, habilidad con las TICs e idiomas. En este sentido, es 
recomendable, disminuir la brecha entre las competencias transversales, logradas por los universitarios y las exigidas por los empleadores, mediante la actualización frecuente del currículo de estudio acorde a las demandas del mercado laboral de una sociedad con cambios disruptivos. Asimismo, fortalecer el aprender a aprender, la mejora continua y la autonomía de los estudiantes.

\section{Referencias}

Álvares, J. (2016). La inserción laboral de los graduados de la Carrera de Administración de $\begin{array}{llll}\text { Empresas } & \text { de UNIANDES. Universitaria. }\end{array}$ http://eduniv.reduniv.edu.cu/index.php?page $=13 \& \mathrm{id}=322 \& \mathrm{db}=1$

Bogers, M., Chesbrough, H., \& Moedas, C. (2018). Open Innovation: Research, Practices, and Polices. California Management Review, $60 \quad$ 5-16. https://doi.org/10.1177/0008125617745086

Bustamante, M., Oyarzún, C., Grandón, M. y Abarza, G. (2015). Fundamentos de la Enseñanza por Competencias a Nivel de Postgrado en dos Universidades Públicas Chilenas Formación Universitaria. 8 (6). http://dx.doi.org/10.4067/S0718-50062015000600004

Hernández, A. I.; Alvarado, J. C. \& Luna, S. M. (2015). Creatividad e innovación: competencias genéricas o transversales en la formación profesional. Revista Virtual Universidad Católica del Norte, 135-151. http://revistavirtual.ucn.edu.co/index.php/RevistaUCN/article/view/620/1155

Iglesias-Cortizas, M. J., \& Rodicio-García, M. L. (2013). El desarrollo de la creatividad e innovación. Un reto ante la crisis actual. Revista de Investigación en Educación, 11(1), 134148. http://reined.webs4.uvigo.es/index.php/reined/article/view/166

Lafontaine, B. (2010). El curriculum universitario por competencias. https://www.academia.edu/4746462/Curriculo_universitario_por_competencias

Llaque, J. P. (2020). Los retos para la gestión y la docencia en la educación superior de América Latina. Review of Global Management, 4(2), 54-63. https://doi.org/10.19083/rgm.v4i2.1140 
Mayorga-Fernández, Madrid-Vivar \& Padial-Díaz (2016). Mercado de trabajo versus formación inicial: competencias del pedagogo en el TFG. Revista Brasileira de Orientação Profissional, 17(2), 125-137. https://www.redalyc.org/pdf/2030/203051246002.pdf

Martínez, P., \& González, N. (2019). El dominio de competencias transversales en Educación Superior en diferentes contextos formativos. Educação e Pesquisa, 45. https://doi.org/10.1590/s1678-4634201945188436

Navarro, E., Jiménez, E., Rappoport, S., Thoillez, B. (2017). Fundamentos de investigación y la innovación educativa. https://www.unir.net/wpcontent/uploads/2017/04/Investigacion_innovacion.pdf

OCDE. (2019). OECD Skills Strategy 2019: skills to shape a better future. OECD, Paris, France.

OCDE. (2012). Better Skills, Better Jobs, Better Lives: A strategic Approach to Skills Policies, OECD Publishing. http://dx.doi.org/10.1787/9789264177338-en

Ordóñez, R. (2010). El cambio, creatividad e innovación. México: Granica S. A.

Pedroza Flores, R. (2018). La universidad 4.0 con currículo inteligente 1.0 en la cuarta revolución industrial. RIDE. Revista Iberoamericana para la Investigación y el Desarrollo Educativo, 9(17), 168-194. https://doi.org/10.23913/ride.v9i17.377.

Pereira, J. (2010). Consideraciones básicas del pensamiento complejo de Edgar Morin, en la educación. Revista Electrónic@ Educare, $\quad$ 14(1), $\quad$ 67-75. https://dialnet.unirioja.es/servlet/articulo?codigo $=4780956$

Piscoya, L (1987). Investigación Científica y Educacional. Lima: Amaru.

Rodríguez, A. (2012). Orientación profesional por competencias transversales para mejorar la competitividad. Universidad de Zaragoza. 